\title{
Medicina de Precisão e suas Mudanças na Oncologia
}

https://doi.org/10.32635/2176-9745.RBC.2019v65n2.412

\author{
Precision Medicine and its Changes in Oncology \\ Medicina de Precisión y sus Cambios en Oncología
}

\section{Alessandra de Sá Earp Siqueira'; Luiz Claudio Santos Thuler²; Letícia Casado³; Anke Bergmann4; Mario Jorge Sobreira da Silva5}

A quarta revolução industrial, também conhecida como Indústria 4.0, vem revolucionando o mundo e traz consequências na área da saúde. Alguns estudiosos já falam sobre uma nova era ${ }^{1}$. A sinergia dos componentes computacionais e físicos, denominada como sistema ciberfísicos, levou ao avanço da implementação da Internet das coisas (do inglês, Internet of Things - IoT), definida como o encontro entre as redes de comunicação humana (exemplificada na Internet) e o mundo real das coisas².

Essas mudanças já chegaram à saúde. Há um número cada vez maior de softwares inteligentes, dispositivos, programas em nuvem, prontuários eletrônicos e aplicativos que monitoram a saúde dos indivíduos e que podem ser integrados a equipamentos médicos e gerar uma massa de dados chamada Big Data, capaz de revolucionar os tratamentos médicos e promover análise em tempo real. Entretanto, essa proliferação das fontes de dados traz um grande desafio que é o de fazer com que esses dados tenham sentido ${ }^{3}$.

O governo americano investiu bilhões de dólares nos últimos anos para construir um database com informações de registros médicos, dados genéticos e diversos dados de saúde de voluntários ${ }^{4}$. O investimento promete customizar os tratamentos médicos, personalizando, até o nível molecular, a verdadeira medicina de precisão. Nesse cenário, o cuidado à saúde deve ser cada vez mais personalizado, baseando-se em resultados de pesquisas com elevado número de pacientes ou no relacionamento de bases de dados públicas existentes, o que se traduz em elevada validade externa.

$\mathrm{O}$ cruzamento de dados das IoT associado às informaçôes dos sistemas intitulados Machine to Machine (M2M) e à computação na nuvem proporcionará a criação de modelos para a identificação, o tratamento e a prevenção à saúde de cada paciente. A expectativa é a criação de novos algoritmos mais eficazes e personalizados que permitirão melhorar a tomada de decisão na aplicação dos recursos públicos nos sistemas de saúde 5 .

Um exemplo dessa revolução na área da oncologia foi a ASCO 2019, cujo tema foi "Cuidar de cada paciente, aprender com cada paciente”. A sessão plenária trouxe dados da Dra. Blythe J. S. Adamson, Ph.D, cientista quantitativa sênior da Flatiron Health, em Nova York, mostrando o uso das informações em tempo real e o seu impacto na expansão do sistema americano de saúde. Dados utilizando Big Data comprovaram a importância do acesso ao sistema americano na redução das disparidades raciais em relação ao tempo até o tratamento do câncer (Abstract LBA1) ${ }^{6}$.

Os Real World Data (RWD) ou Dados de Vida Real, em português, são estudos que coletam informações dos pacientes em geral e, dessa forma, avaliam o impacto de novas tecnologias em saúde. Os RWD utilizam informaçóes de diferentes categorias: 1) clínica, que leva em conta a aderência à tecnologia e as taxas de recidiva da doença e cura na vida real; 2) econômica, que considera os custos associados com os recursos médicos utilizados; e 3) humanista, que dá importância a informaçóes sobre a saúde e a qualidade de vida dos pacientes ${ }^{7}$.

Ainda na ASCO 2019, um excelente exemplo do uso de dados do mundo real foi um estudo de coorte observacional retrospectivo projetado para avaliar as características e os desfechos de pacientes portadores de câncer de pulmão não pequenas células (CPNPC) com ou sem história de doença autoimune. Todos os pacientes foram tratados com inibidores de controle imunológico, em práticas de oncologia predominantemente baseadas na comunidade. O diferencial da pesquisa foi a utilizaçáo dos registros eletrônicos de saúde (do inglês, Eletronic Health Records - EHR) não identificados do banco de dados CancerLinQ ${ }^{\circ}$ da ASCO, explorando os eventos adversos relacionados ao sistema imunológico ${ }^{8}$.

\footnotetext{
1 Editora-Associada da Revista Brasileira de Cancerologia (RBC). Instituto Nacional de Câncer José Alencar Gomes da Silva (INCA). Rio de Janeiro (RJ), Brasil. E-mail: asiqueira@inca.gov.br. Orcid iD: https://orcid.org/0000-0003-3852-7580

${ }_{2}^{2}$ Pesquisador da Epidemiologia Clínica do INCA. Rio de Janeiro, RJ, Brasil. E-mail: Ithuler@inca.gov.br. Orcid iD: https://orcid.org/0000-0003-2550-6537

${ }^{3}$ Editora-Executiva. RBC/INCA. Rio de Janeiro (RJ), Brasil. E-mail: leticiac@inca.gov.br. Orcid iD: https://orcid.org/0000-0001-5962-8765

${ }^{4}$ Editora-Científica. RBC/INCA. Rio de Janeiro (RJ), Brasil. E-mail: abergmann@inca.gov.br. Orcid iD: https://orcid.org/0000-0002-1972-8777

${ }^{5}$ Editor-Associado. RBC/INCA. Rio de Janeiro (RJ), Brasil. E-mail: mario.silva@inca.gov.br. Orcid iD: https://orcid.org/0000-0002-0477-8595
} 
A implantação da medicina de precisão com a utilização de Big Data tem como desafio a segurança e a integridade das informaçōes médicas, e as novas soluções como o Blockchain na saúde parecem ser uma solução promissora. Blockchain tem sido definido como uma tecnologia de registro distribuído a qual visa à descentralização como medida de segurança; ou seja, a criptografia. Além disso, não devem ser ignorados princípios éticos fundamentais como a privacidade, a confidencialidade e a prevenção da ocorrência de danos ao indivíduo?.

$\mathrm{Na}$ saúde mundial, diversos pesquisadores vêm investindo em Big Data, IoT e RWD ${ }^{10}$. No Brasil, por exemplo, segundo o International Data Center (IDC), o mercado das IoT deve movimentar aproximadamente US\$ 13 bilhóes até o próximo ano. Os estudos dessas informaçôes sobre a saúde da população tornam possível a criação de indicadores financeiros, de mortalidade, de morbidade, assim como a promoção da análise de sobrevida. É também possível comparar dados entre sistemas de saúde como o privado e o público do nosso país ${ }^{11}$.

$\mathrm{Na}$ oncologia, a medicina de precisão vem se mostrando promissora. Seu uso permitirá a ampliação do conhecimento na área da pesquisa com dados em tempo real, o que facilitará a disseminação do conhecimento científico e a segurança na introdução de novos fármacos no mercado ${ }^{12}$.

O Brasil dispóe de vários bancos de dados públicos, os quais unem informaçóes que podem ser úteis no desenvolvimento de pesquisas aplicadas à oncologia. Como exemplo, podem ser citados os Registros de Câncer de Base Populacional (RCBP), que fornecem dados de incidência de câncer e vêm sendo implantados no país desde $1967^{13}$. Atualmente, cobrem cerca de 1/4 da população brasileira. Já os Registros Hospitalares de Câncer (RHC) permitem a avaliação da qualidade da assistência prestada ${ }^{14}$. Por meio de um tabulador de dados - o Integrador RHC - é possível acessar informaçôes de mais de três milhôes de casos de câncer atendidos no país nos últimos anos. Por sua vez, o Sistema de Informação sobre Mortalidade (SIM) tem dados disponíveis dos óbitos por câncer desde 1979 e tem sido amplamente utilizado para analisar as tendências de mortalidade nas diferentes topografias da doençç ${ }^{15}$. Há ainda informaçóes sobre exames citopatológicos e mamografias, ambos realizados pelo Sistema Único de Saúde, disponibilizados por meio do Sistema de Informação do Câncer do Colo do Útero (Siscolo) e do Sistema de Informação do Câncer de Mama (Sismama), respectivamente ${ }^{16}$.

A Revista Brasileira de Cancerologia tem publicado estudos utilizando Big Data ao longo dos últimos anos, com o intuito de apoiar as inovaçóes tecnológicas que ampliam o conhecimento científico, especialmente na área da oncologia.

\section{REFERÊNCIAS}

1. Aires RWA, Moreira FK, Freire PS. Indústria 4.0: desafios e tendências para a gestão do conhecimento. In: Santos F, Silva TC, Rochadel W, et al, editors. Anais do $1^{\circ}$ Seminário Internacional Universidades Corporativas e Escolas de Governo - SUCEG; Florianópolis, SC. Florianópolis, SC: Suceg. [acesso 2019 aug. 09]. p. 224-247. Disponível em: http://anais.suceg.ufsc.br/index.php/suceg/article/view/49

2. Atzori L, Iera A, Morabito G. The internet of things: a survey. Comput Networks. 2010;54(15):2787-2805. doi: https:// doi.org/10.1016/j.comnet.2010.05.010

3. Murdoch TB, Detsky AS. The inevitable application of big data to health care. JAMA. 2013;309(13):1351-1352. doi: https://doi.org/10.1001/jama.2013.393

4. Laseri C. Internet das coisas ou IoT: entenda o que é [Internet]. Ada [acesso 2019 aug. 09]. 2018 Mar. 19. Disponível em: https://ada.vc/2018/03/19/internet-das-coisas-ou-iot-entenda-o-que-e/

5. Beam AL, Kohane IS. Big Data and machine learning in health care. JAMA. 2018;319(13):1317-1318. doi: https:// doi.org/10.1001/jama.2017.18391

6. Levitan D. Research highlights aca's effects on access and outcomes in cancer care [Internet]. ASCO Daily News [cited 2019 Aug. 11]. 2019 July 19. Available from: https://dailynews.ascopubs.org/do/10.1200/ADN.19.190365/full/

7. Berger ML, Sox H, Willke RJ, et al. Good practices for real-world data studies of treatment and/or comparative effectiveness: Recommendations from the joint ISPOR-ISPE Special Task Force on real-world evidence in health care decision making. Pharmacoepidemiol Drug Saf. 2017;26(9):1033-1039. doi: https://doi.org/10.1002/pds.4297

8. Donald T. Autoimmune disease and immunotherapy outcomes in patients with NSCLC [Internet]. ASCO Daily News [cited 2019 Aug. 11]. 2019 June 5. Available from: https://dailynews.ascopubs.org/do/10.1200/ADN.19.190344/full/

9. Angraal S, Krumholz HM, Schulz WL. Blockchain technology: applications in health care. Circ Cardiovasc Qual Outcomes. 2017;10(9):e003800. doi: https://doi.org/10.1161/CIRCOUTCOMES.117.003800

10. Adriano P, Simonetto, EO. Indústria 4.0: conceitos e perspectivas para o Brasil. Rev Univ Vale Rio Verde. 2018;16(1). doi: http://dx.doi.org/10.5892/ruvrd.v16i1.4938 
11. Oracle Health Sciences. Medicina de precisão conta com big data para melhorar os tratamentos [Internet]. Saúde Business by Informa Markets [acesso 2019 jul. 24]. 2017 jan. 25. Disponível em: https://saudebusiness.com/voz-damarca/medicina-de-precisao-conta-com-big-data-para-melhorar-os-tratamentos-2/

12. Cabral EJS. Oncologia e internet: como as informaçôes médicas são disponibilizadas para o paciente oncológico no mundo virtual [tese na Internet]. Florianópolis, SC: Universidade Federal de Santa, Centro de Filosofia e Ciências Humanas; 2016. [acesso 2019 jul. 9]. 201 p. Disponível em: https://repositorio.ufsc.br/xmlui/bitstream/ handle/123456789/180880/349168.pdf?sequence $=1$ \&isAllowed $=y$

13. Instituto Nacional de Câncer José Alencar Gomes da Silva. Causas e Prevenção: registros de câncer [Internet]. Rio de Janeiro: INCA; [data desconhecida] - [modificado 2018 ago. 22; acesso 2019 jul. 04]. Disponível em: https: https:// www.inca.gov.br/numeros-de-cancer/registros-de-cancer

14. Instituto Nacional de Câncer. Registros hospitalares de câncer: planejamento e gestão [Internet]. 2. ed. Rio de Janeiro: INCA; 2010 [acesso 2019 ago. 5]. 538 p. Disponível em: https://www.inca.gov.br/numeros-de-cancer/registroshospitalares-de-cancer-rhc

15. Instituto Nacional de Câncer José Alencar Gomes da Silva. Atlas On-line de Mortalidade [Internet]. Rio de Janeiro: INCA; [2014] - [atualizado 2019 maio 30; acesso 2019 jul. 04]. Disponível em: https://mortalidade.inca.gov.br/ MortalidadeWeb/

16. Sistema de Informação do Câncer da Mulher [Internet]. Rio de Janeiro: Datasus; c2008. [acesso 2019 jul. 15]. Disponível em: http://w3.datasus.gov.br/siscam/index.php 